\title{
Paired 2-Disjoint Path Covers and Strongly Hamiltonian Laceability of Bipartite Hypercube-Like Graphs
}

\author{
Shinhaeng Jo ${ }^{\mathrm{a}}$, Jung-Heum Park ${ }^{\mathrm{b}, *}$, Kyung-Yong Chwa ${ }^{\mathrm{a}}$ \\ ${ }^{a}$ Department of Computer Science, KAIST, Daejeon 305-701, Korea \\ ${ }^{b}$ School of Computer Science and Information Engineering, The Catholic University of \\ Korea, Bucheon 420-743, Korea
}

\begin{abstract}
A paired many-to-many $k$-disjoint path cover (paired $k$-DPC for short) of a graph is a set of $k$ vertex-disjoint paths joining $k$ distinct source-sink pairs that altogether cover every vertex of the graph. We consider the problem of constructing paired 2-DPC's in an $m$-dimensional bipartite HL-graph, $X_{m}$, and its application in finding the longest possible paths. It is proved that every $X_{m}, m \geq 4$, has a fault-free paired 2-DPC if there are at most $m-3$ faulty edges and the set of sources and sinks is balanced in the sense that it contains the same number of vertices from each part of the bipartition. Furthermore, every $X_{m}, m \geq 4$, has a paired 2-DPC in which the two paths have the same length if each source-sink pair is balanced. Using 2-DPC properties, we show that every $X_{m}, m \geq 3$, with either at most $m-2$ faulty edges or one faulty vertex and at most $m-3$ faulty edges is strongly Hamiltonian-laceable.
\end{abstract}

Keywords: Disjoint path, strongly Hamiltonian-laceable, Hamiltonian path, bipartite HL-graphs, graph theory, fault tolerance.

\section{Introduction}

Since node and/or link failure is inevitable in a large network, fault tolerance is essential to the network performance. An interconnection network is frequently modeled as a graph, where vertices and edges respectively represent nodes and communication links in the network. The connectivity of

\footnotetext{
* Corresponding author

Email addresses: josh@jupiter.kaist.ac.kr (Shinhaeng Jo), j.h.park@catholic.ac.kr (Jung-Heum Park), kychwa@jupiter.kaist.ac.kr (Kyung-Yong Chwa)
} 
the underlying graph has been a primary measure of fault tolerance [13, 24], and the connectivity of a graph is closely related to the existence of disjoint paths in the graph. Menger's theorem states the connectivity of a graph in terms of the number of disjoint paths of one-to-one type joining a pair of source and sink, whereas the Fan Lemma states the connectivity of a graph in terms of the number of disjoint paths of one-to-many type joining a source to a set of sinks [2]. Moreover, a graph is $k$-connected if and only if it has $k$ disjoint paths of many-to-many type, respectively connecting arbitrary $k$ distinct sources and arbitrary $k$ distinct sinks, where, if a source coincides with a sink, then such source itself is regarded as a path.

One of the central issues in the study of interconnection networks is finding parallel paths, which is naturally related to routing among nodes and fault tolerance of the network [13, 24]. Parallel paths correspond to disjoint paths of the graph. If each copy of a message is routed along a different path of the disjont paths, whichever type they are, then at least one copy eventually arrives at its sink provided the total number of node and link faults is less than the number of disjoint paths. Here, the unique source of one-to-one type and of one-to-many type is assumed to be fault-free, as well as the unique sink of one-to-one type.

A $k$-disjoint path cover ( $k$-DPC for short) of a graph is a set of $k$ (internally) disjoint paths that altogether cover every vertex of the graph. The disjoint path cover problem finds applications in many areas such as software testing, database design, and code optimization $[1,25]$. In addition, the problem is concerned with applications where full utilization of network nodes is important [29]. For example, basic communication problems for the dissemination of information, such as broadcasting (to send a message to all the nodes) and information gathering (to receive a message from each of the nodes), require visiting every node of the network at least once. Since visiting a node more than once results in unnecessary overhead, a disjoint path cover can be employed to avoid this unsatisfactory situation.

Disjoint path is one of the fundamental notions in graph theory from which many properties of a graph can be deduced [2, 24]. Our disjoint path cover problem, in the corresponding optimization version, is to find disjoint paths whose total length is the maximum possible (maxsum). Determining whether there exists a $k$-DPC in general graph was proven to be NP-complete for any fixed $k \geq 1[29,30]$. On the contrary, the shortest disjoint paths problem is to minimize the total length (minsum) or the length of the longest path (minmax). Much attention has been devoted to the shortest disjoint paths problem. For example, refer to [16, 18, 23] etc.

Suppose that there are a set of $k$ sources $S=\left\{s_{1}, s_{2}, \ldots, s_{k}\right\}$ and a set 
of $k \operatorname{sinks} T=\left\{t_{1}, t_{2}, \ldots, t_{k}\right\}$ in a graph $G$ such that $S \cap T=\emptyset$. Sources and sinks are called terminals in general. A many-to-many $k$-disjoint path cover of $G$ joining $S$ and $T$ is a set of $k$ disjoint paths joining sources and sinks. It is called paired if each source $s_{i}$ is joined to a specified sink $t_{i}$. If not, it is called unpaired. The other two possible $k$-disjoint path covers are of one-tomany type joining $S=\{s\}$ and $T=\left\{t_{1}, t_{2}, \ldots, t_{k}\right\}$, and of one-to-one type joining $S=\{s\}$ and $T=\{t\}$, which are clearly understandable. When a graph contains faulty elements, whether vertices or edges, its $k$-disjoint path cover naturally means a $k$-disjoint path cover of the graph with the faulty elements deleted. Some works on the construction of $k$-DPC's in hypercubes $[3,4,7,11]$ and hypercube-like graphs $[15,29,30]$ can be found.

The embedding of a linear array or a ring into an interconnection network can be modeled as finding a long path or cycle, possibly a Hamiltonian path or cycle. A path or cycle in a graph is called Hamiltonian if it contains all the vertices of the graph. This problem has attracted much attention in the literature, such as works on faulty hypercubes $[9,10,17]$. The disjoint path cover problem is closely related to the Hamiltonian problem in that a Hamiltonian path joining a pair of vertices can be viewed as any type of 1-DPC joining them, and a Hamiltonian path joining a pair of vertices that passes through $k-1$ prescribed edges can be obtained directly from some paired $k$-DPC of the graph [29]. For the problem of Hamiltonian paths passing through prescribed edges, see $[6,32]$ for example. Furthermore, disjoint path coverability has been employed to establish some Hamiltonian properties, such as in hypercube-like graphs [21, 27, 29].

A class of hypercube-like interconnection networks, called HL-graphs, was introduced by Vaidya et al. [31]. It gives a unified perspective on many hypercube variants. It is well-known that twisting some edge pairs of a hypercube can reduce diameter while preserving attractive properties. Using this technique, many hypercube variants with smaller diameter have been proposed, such as twisted cube [12], crossed cube [8], and Möbius cube [5]. Most of the networks built in this way are nonbipartite HL-graphs. Furthermore, an interesting subclass of nonbipartite HL-graphs, called restricted HL-graphs, has been proposed and studied in [28, 29, 30].

Bipartite HL-graphs are equitable, that is, the two parts of the bipartition have the same number of vertices. There is a relative paucity of works on bipartite HL-graphs. Some notable studies are mentioned in the following. Every $m$-dimensional bipartite HL-graph $X_{m}, m \geq 2$, has a paired 2-DPC joining $S$ and $T$ if $S$ and $T$ are subsets of different parts [27]. Every $X_{m}, m \geq 2$, possessing at most $m-2$ faulty edges is Hamiltonian-laceable $[21,26]$. An equitable bipartite graph is called Hamiltonian-laceable if each 
pair of vertices contained in different parts is joined by a Hamiltonian path. Recently, Lim et al. [20] presented a vertex-symmetric graph with a small diameter, called bicube, contained in the class of $m$-dimensional bipartite HL-graphs. Its diameter is $\lceil m / 2\rceil$, which is one of the smallest among the hypercube variants, thus providing further motivation for the study of bipartite HL-graphs.

In this paper, we investigate the problem of constructing paired 2-disjoint path covers in bipartite HL-graphs. It will be shown that every $m$-dimensional bipartite HL-graph $X_{m}, m \geq 4$, with $m-3$ or less faulty edges has a paired 2-DPC joining $S$ and $T$ provided $S \cup T$ is balanced. A vertex subset of an equitable bipartite graph is balanced if it contains the same number of vertices from each part. For an equitable bipartite graph to have a paired 2-DPC, it is necessary that $S \cup T$ is balanced. Furthermore, every $X_{m}$, $m \geq 4$, has a paired 2-DPC joining $S$ and $T$ in which the two paths are of the same length if $S$ and $T$ are subsets of different parts.

Using the result on paired 2-DPC, we will derive strongly Hamiltonianlaceability of bipartite HL-graphs. An equitable bipartite graph is strongly Hamiltonian-laceable if it is Hamiltonian-laceable and each pair of vertices in the same part is joined by a path of length $n-2$, where $n$ is the number of vertices in the graph. We will show that every $X_{m}$ with fault set $F$ is strongly Hamiltonian-laceable if either $F$ contains at most $m-2$ edges or $F$ contains at most $m-3$ edges and one vertex. The definition of strongly Hamiltonian-laceability of an inequitable bipartite graph is provided in the next section.

In the rest of this paper, we use standard terminology in graph theory (see ref. [2]). This paper is organized as follows. In the next section, we present some definitions and related work. The paired 2-disjoint path cover problems are investigated in Section 3, and strongly Hamiltonian-laceability is derived in Section 4. Finally, we give concluding remarks in Section 5.

\section{Definitions and Related Work}

For graphs $G_{0}$ and $G_{1}$ having the same number of vertices, we denote by $G_{0} \oplus G_{1}$ an arbitrary graph whose vertex set is $V\left(G_{0}\right) \cup V\left(G_{1}\right)$ and whose edge set is $E\left(G_{0}\right) \cup E\left(G_{1}\right) \cup E_{2}$, where $E_{2}=\left\{(u, \phi(u)): u \in V\left(G_{0}\right), \phi: V\left(G_{0}\right) \rightarrow\right.$ $V\left(G_{1}\right)$ is a bijection $\}$. Here, $V(G)$ and $E(G)$ denote the vertex set and edge set of a graph $G$, respectively. The class of HL-graphs can be defined by repeatedly applying the $\oplus$ operation as follows: $H L_{0}=\left\{K_{1}\right\}$, where $K_{1}$ is the trivial graph; for $m \geq 1, H L_{m}=\left\{G_{0} \oplus G_{1}: G_{0}, G_{1} \in H L_{m-1}\right\}$. A 
graph contained in $H L_{m}$ is called an m-dimensional $H L$-graph. Every $m$ dimensional HL-graph has $2^{m}$ vertices and is of degree $m$. This paper deals with bipartite HL-graphs, which are equitable by definition.

Let $G$ be a bipartite graph. For convenience, we refer to vertices of one part of the bipartition as black and the vertices of the other part as white. We denote by $c(v)$ the color of the vertex $v$. Let $G$ have $n_{b}$ black vertices and $n_{w}$ white vertices, and let $n=n_{b}+n_{w}$. Strongly Hamiltonian-laceability of a bipartite graph is defined in terms of $L^{\mathrm{OPT}}$-path. If $n_{b}=n_{w}$, a path of length $n-1$ joining a balanced pair of vertices is called an $L^{\mathrm{OPT}}$-path; for a pair of vertices with the same color, a path of length $n-2$ is called an $L^{\mathrm{OPT}}$-path. If $n_{b}<n_{w}$, the length of an $L^{\mathrm{OPT}}$-path is $2 n_{b}$ for a pair of white vertices, $2 n_{b}-1$ for a balanced pair of vertices, and $2 n_{b}-2$ for a pair of black vertices. If $n_{w}<n_{b}$, an $L^{\mathrm{OPT}}$-path is defined symmetrically.

A bipartite graph $G$ is called strongly Hamiltonian-laceable if every pair of vertices is joined by an $L^{\mathrm{OPT}}$-path. The graph $G$ is called $f$-edge-fault strongly Hamiltonian-laceable if $G \backslash F$ is strongly Hamiltonian-laceable for any edge-fault set $F$ with $|F| \leq f$. The graph $G$ is called $f_{v}$-vertex-fault and $f_{e}$-edge-fault strongly Hamiltonian-laceable if $G \backslash\left(F_{v} \cup F_{e}\right)$ is strongly Hamiltonian-laceable for any vertex-fault set $F_{v}$ with $\left|F_{v}\right| \leq f_{v}$ and any edge-fault set $F_{e}$ with $\left|F_{e}\right| \leq f_{e}$.

Throughout this paper, let $F$ denote a set of faulty elements (vertices and/or edges), which corresponds to the set of node and/or link failures. A path in a graph is represented as a sequence of vertices. An $s$ - $t$ path refers to a path from vertex $s$ to $t$. Let $P$ be an $s$ - $t$ path and $u$ be a vertex on $P$. We denote by $\operatorname{prev}_{P}(u)$ and $\operatorname{next}_{P}(u)$ the vertices adjacent to $u$ on $P$ encountered just before and just after $u$, respectively, when we traverse $P$ starting at $s$. Of course, $\operatorname{prev}_{P}(s)$ and $\operatorname{next}_{P}(t)$ are undefined.

A paired 2-DPC problem in bipartite HL-graphs without faults for balanced source-sink pairs was studied by Park et al. [27], as stated in Lemma 1. This lemma will be extended to Theorem 1 of the next section in that sourcesink pairs may have the same color and a bounded number of faulty edges are allowed. $X_{m}$ denotes an $m$-dimensional bipartite HL-graph.

Lemma 1. [27] Every $X_{m}, m \geq 2$, has a paired 2-DPC joining $S=\left\{s_{1}, s_{2}\right\}$ and $T=\left\{t_{1}, t_{2}\right\}$ if $S$ and $T$ are subsets of different parts of the bipartition.

It is known that every $X_{m}$ is Hamiltonian-laceable [27] and every $X_{m}$, $m \geq 3$, is $(m-3)$-edge-fault Hamiltonian-laceable [14]. Hamiltonian-laceability of $X_{m}$ with at most $m-2$ faulty edges was studied independently by Park [26] and by Lin et al. [21], as shown in Lemma 2. In addition, a Hamiltonian property of $X_{m}$ in the presence of a single vertex fault was reported 
in $[22,26]$, as shown in Lemma 3 . We extend these two lemmas to the case that there exist at most one single vertex fault and at most $m-3$ faults in total, as will be shown in Theorem 3 of Section 4 .

Lemma 2. [21, 26] Every $X_{m}, m \geq 2$, is $(m-2)$-edge-fault Hamiltonianlaceable.

Lemma 3. [22, 26] There exists an s-t Hamiltonian path in $X_{m} \backslash u$ for any distinct vertices $s, t$, and $u$ such that $c(s)=c(t) \neq c(u)$.

\section{Paired 2-Disjoint Path Covers in Bipartite HL-graphs}

In this section, we consider a paired 2-DPC problem in bipartite HLgraphs. We show that every $X_{m}, m \geq 4$, with at most $m-3$ faulty edges has a paired 2-DPC joining $S$ and $T$ for given balanced $S \cup T$, and that every $X_{m}, m \geq 4$, has a paired 2 -DPC in which the two paths, $s_{1}-t_{1}$ path and $s_{2}-t_{2}$ path, have the same length provided $S$ and $T$ are contained in different parts. The latter type of paired 2-DPC is known as a 2-equaldisjoint path cover (2-eq-DPC for short) and studied by Lai et al. [19] in some nonbipartite hypercube-like graphs.

In a graph $G_{0} \oplus G_{1}$, a vertex $u$ of $G_{i}$ has a unique neighbor in $G_{1-i}$. We denote it by $\bar{u}$ and call it a mate of $u$. We call an edge between a vertex and its mate a bridge. $F_{0}$ and $F_{1}$ denote the sets of faulty elements in $G_{0}$ and $G_{1}$, respectively, and $F_{2}$ denotes the set of faulty bridges, so that $F=F_{0} \cup F_{1} \cup F_{2}$. Recall $F$ denotes the set of faulty elements. Hereafter in this paper, we denote by 2 -DPC $\left[\left(s_{1}, t_{1}\right),\left(s_{2}, t_{2}\right) \mid G, F\right]$ a paired 2 -DPC in a graph $G$ with a fault set $F$ joining a set of sources $S=\left\{s_{1}, s_{2}\right\}$ and a set of sinks $T=\left\{t_{1}, t_{2}\right\}$ such that $S \cap T=\emptyset$.

\subsection{Paired 2-DPC in $X_{m}$ with at most $m-3$ faulty edges}

It is assumed that $S \cup T$ is balanced, where $S=\left\{s_{1}, s_{2}\right\}$ and $T=\left\{t_{1}, t_{2}\right\}$. We begin by considering a paired 2-DPC problem in the 3 -dimensional bipartite HL-graph, which is isomorphic to the 3-dimensional hypercube $Q_{3}$. By Lemma 1, $Q_{3}$ has a paired 2-DPC joining $S$ and $T$ if each source-sink pair is balanced. If not, then $\left\{s_{1}, t_{1}\right\}$ is contained in one part of the bipartition and is unique up to symmetry. There are two positions of $\left\{s_{2}, t_{2}\right\}$ such that no paired 2-DPC joining $S$ and $T$ exists, as shown in Fig. 1. There are only two forbidden configurations that do not allow any paired 2-DPC as proved below. We denote by $N_{G}(u)$, or just by $N(u)$ if no confusion can arise, the set of vertices adjacent to $u$ in a graph $G$. 


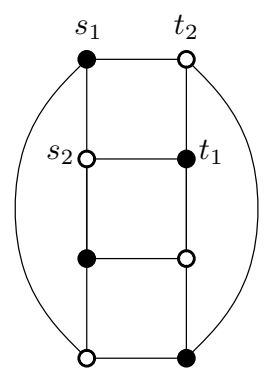

(a)

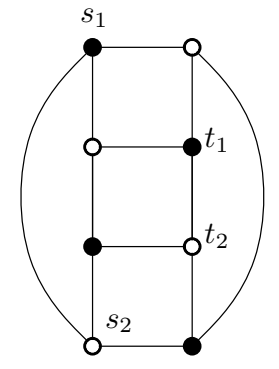

(b)

Figure 1: Forbidden configurations that do not allow any paired 2-DPC.

Lemma 4. Let $\left\{s_{1}, t_{1}\right\}$ be a subset of one part of the bipartition of $Q_{3}$. Then, there exists a paired 2-DPC joining $S$ and $T$ if and only if $\left\{s_{2}, t_{2}\right\}$ is contained in the other part (so, $S \cup T$ is balanced) and it is equal to neither $N\left(s_{1}\right) \cap N\left(t_{1}\right)$ nor $N\left(s_{1}\right)$ XOR $N\left(t_{1}\right)$, where $N\left(s_{1}\right)$ xOR $N\left(t_{1}\right)=$ $\left(N\left(s_{1}\right) \cup N\left(t_{1}\right)\right) \backslash\left(N\left(s_{1}\right) \cap N\left(t_{1}\right)\right)$.

Proof. For a fixed $\left\{s_{1}, t_{1}\right\}$, there are $\left(\begin{array}{l}4 \\ 2\end{array}\right)=6$ possible positions of $\left\{s_{2}, t_{2}\right\}$. It can be easily verified that there exist four positions of $\left\{s_{2}, t_{2}\right\}$ (excluding the two forbidden configurations) that allow a paired 2-DPC joining $\mathrm{S}$ and T.

A vertex $u$ is called free if $u$ is fault-free and not a terminal. An edge $(u, v)$ is called free if it is not faulty and both $u$ and $v$ are free. For a terminal $x \in V\left(G_{i}\right)$, we denote by $x^{*}$ an arbitrary vertex in $R(x)$, where $R(x)=\left\{y: y \in V\left(G_{1-i}\right), c(y)=c(x)\right.$, and $(y, \bar{y})$ is free $\}$. We call $x^{*}$ a pseudo-terminal of $x$.

Theorem 1. Every $m$-dimensional bipartite $H L$-graph $X_{m}, m \geq 4$, with $m-3$ or less faulty edges has a paired 2-DPC joining $S$ and $T$ provided $S \cup T$ is balanced.

Proof. The proof will proceed by induction on $m$. Let $X_{m}=G_{0} \oplus G_{1}$, where each $G_{i}, i=0,1$, is isomorphic to an $(m-1)$-dimensional bipartite HL-graph. Keep in mind that each $G_{i}$ has $2^{m-1}$ vertices of degree $m-1$ and is an equitable bipartite graph. The proof for the basis, i.e., when $m=4$, is similar to that for the induction step, i.e., when $m \geq 5$, but there are additional subtleties. We combine these proofs to avoid repetition. For $m \geq 5$, we will assume that each $G_{i}$ has a paired 2-DPC joining balanced terminals provided the number of faulty edges is at most $m-4$. For $m=4$, 
we can assume the same, except forbidden configurations, by Lemmas 1 and 4. Since a 2 -DPC in $X_{m}$ with a virtual fault set $F \cup F^{\prime}$, where $F^{\prime}$ is a set of arbitrary $m-3-|F|$ fault-free edges, is also a 2-DPC in $X_{m}$ with the fault set $F$, we can assume that $|F|=m-3$. It is assumed w.l.o.g. that the number of terminals in $G_{0}$ is greater than or equal to that in $G_{1}$. There are four cases depending on the positions of terminals.

Case 1: $s_{1}, t_{1} \in V\left(G_{0}\right)$ and $s_{2}, t_{2} \in V\left(G_{1}\right)$. (See Fig. 2a.)

If $c\left(s_{1}\right) \neq c\left(t_{1}\right)$, an $s_{1}-t_{1}$ Hamiltonian path in $G_{0}$ and an $s_{2}-t_{2}$ Hamiltonian path in $G_{1}$ constitute a paired 2-DPC, which is what we want. The existence of the two Hamiltonian paths relies on Lemma 2. Now, assume that $c\left(s_{1}\right)=$ $c\left(t_{1}\right)$. W.l.o.g., we assume $\left|F_{0}\right| \geq\left|F_{1}\right|$. Then, $\left|F_{1}\right| \leq\lfloor(m-3) / 2\rfloor \leq m-4$ for every $m \geq 4$. We claim that it is possible to select two pseudo-terminals $s_{2}^{*}$ and $t_{2}^{*}$ in $G_{0}$ so that there exists a $2-\mathrm{DPC}\left[\left(s_{1}, t_{1}\right),\left(s_{2}^{*}, t_{2}^{*}\right) \mid G_{0}, F_{0}\right]$. The proof of this claim is deferred for a moment. Suppose that the claim holds true. Then there exists a 2 -DPC $\left[\left(s_{2}, \bar{s}_{2}^{*}\right),\left(t_{2}, \bar{t}_{2}^{*}\right) \mid G_{1}, F_{1}\right]$ by induction hypothesis when $m \geq 5$ and by Lemma 1 when $m=4$. To obtain a paired 2-DPC in $X_{m}$, it suffices to merge the two 2-DPC's with bridges $\left(s_{2}^{*}, \bar{s}_{2}^{*}\right)$ and $\left(t_{2}^{*}, \bar{t}_{2}^{*}\right)$.

It remains to prove the claim. For the first case, let $\left|F_{0}\right| \leq m-4$. The number of candidates for $s_{2}^{*}$ and $t_{2}^{*}$ is at least $2^{m-2}-\left|F_{2}\right| \geq 2^{m-2}-(m-3) \geq 3$ for any $m \geq 4$, and thus picking up arbitrary two candidates is sufficient when $m \geq 5$. When $m=4$, a care should be taken since arbitrary candidates may not allow a paired 2-DPC in $G_{0}$ since $c\left(s_{1}\right)=c\left(t_{1}\right) \neq c\left(s_{2}\right)=c\left(t_{2}\right)$. We have $|F|=\left|F_{2}\right|=1$. To avoid forming a forbidden configuration, it suffices to pick up one in $N\left(s_{1}\right) \cap N\left(t_{1}\right)$ and the other in $N\left(s_{1}\right)$ xor $N\left(t_{1}\right)$ by Lemma 4 . Furthermore, we can assume both $\left(s_{2}^{*}, \bar{s}_{2}^{*}\right)$ and $\left(t_{2}^{*}, \bar{t}_{2}^{*}\right)$ are fault-free since both $N\left(s_{1}\right) \cap N\left(t_{1}\right)$ and $N\left(s_{1}\right)$ XoR $N\left(t_{1}\right)$ have two vertices. For the second case, assume that $\left|F_{0}\right|=m-3$ and $\left|F_{1}\right|=\left|F_{2}\right|=0$. We can pick up pseudo-terminal $t_{2}^{*}$ such that $\left(t_{2}^{*}, t_{1}\right) \notin E\left(G_{0}\right)$ since $2^{m-2}>m-1$ for every $m \geq 4$. There exists an $s_{1}-t_{2}^{*}$ Hamiltonian path in $G_{0}$ by Lemma 2 . We set $s_{2}^{*}=\operatorname{next}_{P}\left(t_{1}\right)$. Clearly, $s_{2}^{*} \neq t_{2}^{*}$. If we remove $\left(t_{1}, s_{2}^{*}\right)$ from $P$, there remain an $s_{1}-t_{1}$ path and an $s_{2}^{*}-t_{2}^{*}$ path. These two paths are vertex-disjoint and cover all the vertices of $G_{0}$. Thus, the claim is proved.

Case 2: $s_{1}, s_{2} \in V\left(G_{0}\right)$ and $t_{1}, t_{2} \in V\left(G_{1}\right)$. (See Fig. 2b.)

Without loss of generality, we assume $\left|F_{0}\right| \geq\left|F_{1}\right|$. Similar to the proof of Case 1 , we claim that there exist two pseudo-terminals $t_{1}^{*}$ and $t_{2}^{*}$ such that there exists a 2 -DPC $\left[\left(s_{1}, t_{1}^{*}\right),\left(s_{2}, t_{2}^{*}\right) \mid G_{0}, F_{0}\right]$. Provided the claim holds true, we find a $2-\mathrm{DPC}\left[\left(\bar{t}_{1}^{*}, t_{1}\right),\left(\overline{t_{2}^{*}}, t_{2}\right) \mid G_{1}, F_{1}\right]$ and then merge the two DPC's to obtain a desired 2-DPC. Since $c\left(\bar{t}_{1}^{*}\right) \neq c\left(t_{1}\right)$ and $c\left(\bar{t}_{2}^{*}\right) \neq c\left(t_{2}\right)$, the 2-DPC in $G_{1}$ always exists by induction hypothesis and Lemma 1 .

Let us prove the claim. Suppose $\left|F_{0}\right| \leq m-4$ for the first case. If either 
$m \geq 5$ or both $m=4$ and $c\left(s_{1}\right) \neq c\left(t_{1}\right)$, then picking up arbitrary pseudoterminals is sufficient. In this case, the number of candidates for each of $t_{1}^{*}$ and $t_{2}^{*}$ is at least $2^{m-2}-(m-3)-2 \geq 1$ for any $m \geq 4$. Now, assume that $m=4$ and $c\left(s_{1}\right)=c\left(t_{1}\right)$, where $|F|=\left|F_{2}\right|=1$. To avoid forbidden configurations, we will select pseudo-terminals so that the subgraph of $G_{0}$ induced by terminals and pseudo-terminals is isomorphic to a path of length three. Note that the subgraph induced by four terminals in a forbidden configuration is isomorphic to a cycle of length four (Fig. 1a) or a graph consisting of two paths of length one each (Fig. 1b).

If $\left(s_{1}, s_{2}\right) \notin E\left(G_{0}\right)$, there exists a vertex $x \in N\left(s_{2}\right)$ such that $x \in R\left(t_{1}\right)$ and $y \in R\left(t_{2}\right)$ for some $y \in N(x) \cap N\left(s_{1}\right)$. Assigning $x$ and $y$ to $t_{1}^{*}$ and $t_{2}^{*}$, respectively, results in an induced path $\left(s_{1}, t_{2}^{*}, t_{1}^{*}, s_{2}\right)$ since $s_{1}$ and $s_{2}$ are not adjacent. The case when $\left(s_{1}, s_{2}\right) \in E\left(G_{0}\right)$ remains to be considered. Let $X=N\left(s_{2}\right) \backslash s_{1}$ and $Y=N\left(s_{1}\right) \backslash s_{2}$. Let $\left\{z_{1}, z_{2}\right\}=V\left(G_{0}\right) \backslash\left(N\left(s_{1}\right) \cup N\left(s_{2}\right)\right)$ with $c\left(z_{1}\right)=c\left(s_{1}\right)$. Then, $z_{1}$ and $z_{2}$ are adjacent to every element of $Y$ and $X$, respectively. Observe that (i) $R\left(t_{1}\right) \subseteq X \cup\left\{z_{1}\right\}, R\left(t_{2}\right) \subseteq Y \cup\left\{z_{2}\right\}$, and (ii) $\left|R\left(t_{1}\right)\right|,\left|R\left(t_{2}\right)\right| \geq 1,\left|R\left(t_{1}\right)\right|+\left|R\left(t_{2}\right)\right| \geq 3$. If $X \cap R\left(t_{1}\right)=\emptyset$, then for some $t_{2}^{*} \in Y,\left(t_{1}^{*}, t_{2}^{*}, s_{1}, s_{2}\right)$ forms an induced path, where $t_{1}^{*}=z_{1}$. A symmetric argument works when $Y \cap R\left(t_{2}\right)=\emptyset$. Now, assume $X \cap R\left(t_{1}\right), Y \cap R\left(t_{2}\right) \neq$ $\emptyset$. If $X \subseteq R\left(t_{1}\right)$, then for some $t_{2}^{*} \in Y$, there exists $t_{1}^{*} \in X$ such that $\left(t_{2}^{*}, t_{1}^{*}\right) \notin E\left(G_{0}\right)$ and thus we have an induced path $\left(t_{2}^{*}, s_{1}, s_{2}, t_{1}^{*}\right)$. In a symmetric manner, we can also construct an induced path $\left(t_{2}^{*}, s_{1}, s_{2}, t_{1}^{*}\right)$ when $Y \subseteq R\left(t_{2}\right)$. Finally, suppose $\left|X \cap R\left(t_{1}\right)\right|=\left|Y \cap R\left(t_{2}\right)\right|=1$. At least one of $z_{1}$ and $z_{2}$, say $z_{1}$ is contained in $R\left(t_{1}\right) \cup R\left(t_{2}\right)$. Then, we have an induced path $\left(t_{1}^{*}, t_{2}^{*}, s_{1}, s_{2}\right)$, where $t_{1}^{*}=z_{1}$ and $t_{2}^{*} \in Y \cap R\left(t_{2}\right)$.

For the remaining case, let $\left|F_{0}\right|=m-3$ and $\left|F_{1}\right|=\left|F_{2}\right|=0$. If $c\left(s_{1}\right) \neq$ $c\left(s_{2}\right)$, then there exists an $s_{1}-s_{2}$ Hamiltonian path $P$ in $G_{0}$. We show that there exists an edge $(u, v)$ with $u=\operatorname{prev}_{P}(v)$ on $P$ such that (i) $c(u)=c\left(t_{1}\right)$ and (ii) both $\bar{u}$ and $\bar{v}$ are free. There are at least $2^{m-2}-1$ candidate edges satisfying (i), and there are two blocking elements, i.e. terminals in $G_{1}$. Since $2^{m-2}-1 \geq 3$ for any $m \geq 4$, there exists at least one such edge $(u, v)$. For our purpose, it suffices to remove $(u, v)$ from $P$ and set $t_{1}^{*}=u$ and $t_{2}^{*}=v$. If $c\left(s_{1}\right)=c\left(s_{2}\right)$, we pick up a pseudo-terminal $t_{2}^{*}$ in $G_{0}$ and find an $s_{1}-t_{2}^{*}$ Hamiltonian path $P$ in $G_{0}$. It suffices to set $t_{1}^{*}=\operatorname{prev}_{P}\left(s_{2}\right)$ and remove $\left(t_{1}^{*}, s_{2}\right)$ from $P$. Obviously, $c\left(t_{1}^{*}\right)=c\left(t_{1}\right)$ and $\overline{t_{1}^{*}} \neq t_{1}, t_{2}$. The claim is proved.

Case 3: $s_{1}, t_{1}, s_{2} \in V\left(G_{0}\right)$ and $t_{2} \in V\left(G_{1}\right)$. (See Fig. 2c.)

We claim that (i) for some pseudo-terminal $t_{2}^{*}$, there exists a $2-\operatorname{DPC}\left[\left(s_{1}, t_{1}\right)\right.$, $\left.\left(s_{2}, t_{2}^{*}\right) \mid G_{0}, F_{0}\right]$, or (ii) $G_{0} \backslash\left(F_{0} \cup\left\{s_{2}\right\}\right)$ has an $s_{1}-t_{1}$ Hamiltonian path, $\left(s_{2}, \overline{s_{2}}\right)$ is fault-free, and $c\left(\overline{s_{2}}\right) \neq c\left(t_{2}\right)$. Provided the claim holds true, a paired 2 - 
DPC in $X_{m}$ is obtained by merging the 2 -DPC of $G_{0}$ and a $\overline{t_{2}^{*}}-t_{2}$ Hamiltonian path in $G_{1}$ if (i) is satisfied, or by merging the $s_{1}-t_{1}$ Hamiltonian path of $G_{0}$ and an $\overline{s_{2}}-t_{2}$ Hamiltonian path in $G_{1}$ if (ii) is satisfied.

To prove the claim, assume $\left|F_{0}\right| \leq m-4$ first. It is possible to select $t_{2}^{*}$ satisfying (i) as follows. When either $m \geq 5$ or both $m=4$ and $c\left(s_{1}\right) \neq c\left(t_{1}\right)$, it is sufficient to pick up an arbitrary vertex in $R\left(t_{2}\right)$. When $m=4$ and $c\left(s_{1}\right)=c\left(t_{1}\right)$, as shown in Fig. 1, there are two choices to avoid forming a forbidden configuration and anyone of them can be selected as $t_{2}^{*}$. Now, we assume $\left|F_{0}\right|=m-3$. Without loss of generality, we can assume that $c\left(s_{1}\right) \neq c\left(s_{2}\right)$. Then, there exists an $s_{1}-s_{2}$ Hamiltonian path $P$ in $G_{0}$. If $\operatorname{next}_{P}\left(t_{1}\right) \neq s_{2}$, it suffices to set $t_{2}^{*}=\operatorname{next}_{P}\left(t_{1}\right)$ and remove $\left(t_{1}, t_{2}^{*}\right)$ from $P$. Certainly, $c\left(t_{2}^{*}\right)=c\left(t_{2}\right)$ and $\overline{t_{2}^{*}} \neq t_{2}$. Thus, (i) is satisfied. If $\operatorname{next}_{P}\left(t_{1}\right)=s_{2}$, removing $\left(t_{1}, s_{2}\right)$ from $P$ results in an $s_{1}$ - $t_{1}$ Hamiltonian path in $G_{0} \backslash\left(F_{0} \cup\right.$ $\left.\left\{s_{2}\right\}\right)$. Furthermore, $\left(s_{2}, \overline{s_{2}}\right)$ is fault-free and $c\left(\overline{s_{2}}\right) \neq c\left(t_{2}\right)$. Thus, (ii) is satisfied and the claim is proved.

Case 4: $s_{1}, t_{1}, s_{2}, t_{2} \in V\left(G_{0}\right)$. (See Fig. 2 d.)

Suppose that either $m \geq 5$ or $m=4$ and terminals do not form a forbidden configuration in $G_{0}$. We let $F_{0}^{\prime}=F_{0}$ if $\left|F_{0}\right| \leq m-4$; otherwise, let $F_{0}^{\prime}=F_{0} \backslash e$ for an arbitrary faulty edge $e$ in $G_{0}$. Then, $\left|F_{0}^{\prime}\right| \leq m-4$. So, there exists a paired 2-DPC in $G_{0} \backslash F_{0}^{\prime}$ joining $S$ and $T$. If no path in the DPC passes through $e$, let $(u, v)$ be an edge on a path in the DPC such that $(u, \bar{u})$ and $(v, \bar{v})$ are fault-free; otherwise, let $(u, v)=e$. To obtain a paired 2-DPC in $X_{m}$, it suffices to replace $(u, v)$ with a path $\left(u, P_{1}, v\right)$, where $P_{1}$ is an $\bar{u}-\bar{v}$ Hamiltonian path in $G_{1}$.

Finally, let $m=4$ and terminals form a forbidden configuration. Recall $|F|=1$. There are two forbidden configurations up to symmetry by Lemma 4. For the forbidden configuration of $\left\{s_{2}, t_{2}\right\}=N\left(s_{1}\right) \cap N\left(t_{1}\right)$, as shown in Fig. 2e, let $w_{1}$ and $w_{2}$ be vertices in $N\left(s_{1}\right)$ xoR $N\left(t_{1}\right)$ and let $b_{1}$ and $b_{2}$ be vertices in $N\left(s_{2}\right)$ xor $N\left(t_{2}\right)$. We denote by $P[u, v]$ (resp. $P_{h}[u, v]$ ) an $u-v$ path (resp. an $u-v$ Hamiltonian path) in $G_{1}$. If $F_{0} \cup F_{2}=\emptyset$, there are two paths $\left(s_{1}, w_{2}, P_{h}\left[\bar{w}_{2}, \overline{t_{1}}\right], t_{1}\right)$ and $\left(s_{2}, b_{2}, w_{1}, b_{1}, t_{2}\right)$, which form a paired 2 -DPC in $X_{m}$. If $F_{1}=\emptyset$, we let

$$
\begin{aligned}
& P_{1}=\left(s_{1}, P\left[\overline{s_{1}}, \bar{w}_{2}\right], w_{2}, b_{2}, w_{1}, t_{1}\right), P_{2}=\left(s_{2}, P\left[\overline{s_{2}}, \overline{b_{1}}\right], b_{1}, t_{2}\right), \\
& P_{1}^{\prime}=\left(s_{1}, w_{2}, b_{1}, w_{1}, P\left[\bar{w}_{1}, \overline{t_{1}}\right], t_{1}\right), P_{2}^{\prime}=\left(s_{2}, b_{2}, P\left[\overline{b_{2}}, \overline{t_{2}}\right], t_{2}\right),
\end{aligned}
$$

where $\left\{P\left[\overline{s_{1}}, \overline{w_{2}}\right], P\left[\overline{s_{2}}, \overline{b_{1}}\right]\right\}$ and $\left\{P\left[\overline{w_{1}}, \overline{t_{1}}\right], P\left[\overline{b_{2}}, \overline{t_{2}}\right]\right\}$ are 2 -DPC's in $G_{1}$. Such 2-DPC's exist by Lemma 1 . Since $\left\{P_{1}, P_{2}\right\}$ and $\left\{P_{1}^{\prime}, P_{2}^{\prime}\right\}$ share neither bridges nor edges of $G_{0}$, at least one of the two sets is a desired 2-DPC in $X_{m}$.

For the forbidden configuration of $\left\{s_{2}, t_{2}\right\}=N\left(s_{1}\right)$ xOR $N\left(t_{1}\right)$, as shown in Fig. 2f, we let $w_{1}$ and $w_{2}$ be vertices in $N\left(s_{1}\right) \cap N\left(t_{1}\right)$ and let $b_{1}$ and $b_{2}$ 


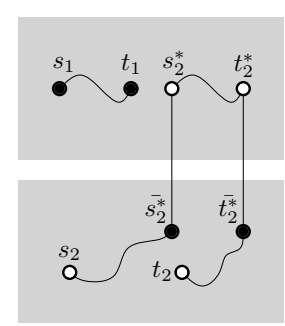

(a) Case 1

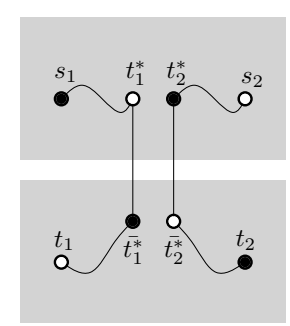

(b) Case 2

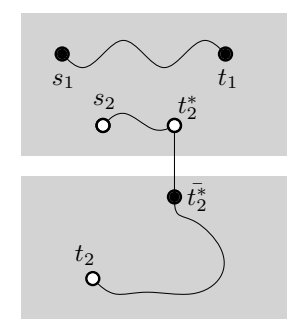

(c) Case 3

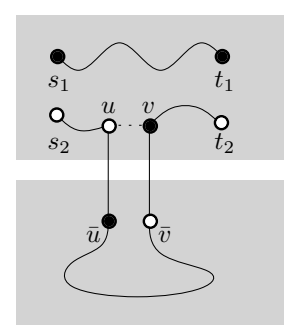

(d) Case 4
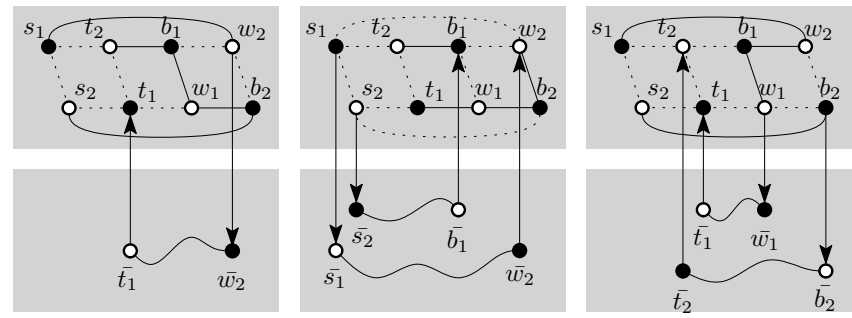

(e) $\left\{s_{2}, t_{2}\right\}=N\left(s_{1}\right) \cap N\left(t_{1}\right)$
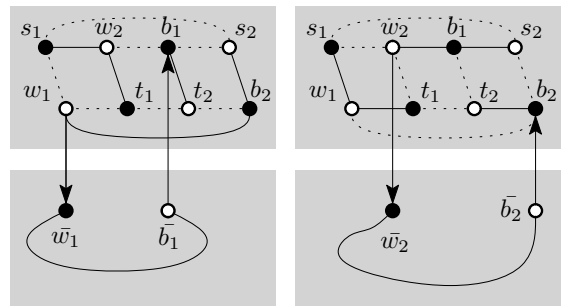

(f) $\left\{s_{2}, t_{2}\right\}=N\left(s_{1}\right)$ xOR $N\left(t_{1}\right)$.

Figure 2: Illustration of the proof of Theorem 1.

be vertices in $N\left(s_{2}\right) \cap N\left(t_{2}\right)$. We let

$$
\begin{aligned}
& P_{1}=\left(s_{1}, w_{2}, t_{1}\right), P_{2}=\left(s_{2}, b_{2}, w_{1}, P_{h}\left[\bar{w}_{1}, \overline{b_{1}}\right], b_{1}, t_{2}\right), \\
& P_{1}^{\prime}=\left(s_{1}, w_{1}, t_{1}\right), P_{2}^{\prime}=\left(s_{2}, b_{1}, w_{2}, P_{h}\left[\bar{w}_{2}, \overline{b_{2}}\right], b_{2}, t_{2}\right) .
\end{aligned}
$$

Similar to the proof of the previous case, we can conclude at least one of $\left\{P_{1}, P_{2}\right\}$ and $\left\{P_{1}^{\prime}, P_{2}^{\prime}\right\}$ is a paired 2-DPC in $X_{m}$. This completes the proof of Theorem 1.

Theorem 1 is an extension of the work stated in Lemma 1 by Park et al. in [27]. The number $m-3$ of faulty edges is the maximum possible in the sense that no $m$-dimensional bipartite HL-graph with $m-2$ faulty edges is guaranteed to have a paired 2-DPC joining $S$ and $T$ even if $S \cup T$ is balanced. Imagine the situation that $s_{2}, t_{2} \in N\left(s_{1}\right)$ and all the edges that are incident to $s_{1}$ but not to $s_{2}$ or $t_{2}$ are faulty. The number of faulty edges is $m-2$. For any $t_{1}$ with $c\left(t_{1}\right)=c\left(s_{1}\right), S \cup T$ is balanced. However, no 
paired 2-DPC joining $S$ and $T$ exists. The characterization of the existence of paired 2-DPC in $X_{m}$ with $m-2$ or more faulty edges is an open problem.

\subsection{Paired 2-eq-DPC in fault-free $X_{m}$}

In an $m$-dimensional bipartite HL-graph $X_{m}$ without faults, we consider the problem of constructing 2-eq-DPC's. It is necessary for $X_{m}$ to have a paired 2-eq-DPC that $c\left(s_{1}\right) \neq c\left(t_{1}\right)$ and $c\left(s_{2}\right) \neq c\left(t_{2}\right)$. The reason for this is that each path of a paired 2 -eq-DPC in $X_{m}$ should contain $2^{m-1}$ vertices. It will be shown that $X_{m}, m \geq 4$, has a 2 -eq-DPC if each sourcesink pair is balanced. We begin by considering the 2-eq-DPC problem in the 3 -dimensional bipartite HL-graph $Q_{3}$. $Q_{3}$ may not have a 2-eq-DPC even if each source-sink pair is balanced. A necessary and sufficient condition for $Q_{3}$ to have a 2 -eq-DPC is derived as follows.

Lemma 5. Suppose that $S$ and $T$ are subsets of different parts of the bipartition of $Q_{3}$. Then, $Q_{3}$ has a paired 2-eq-DPC joining $S$ and $T$ if and only if $\left(s_{1}, t_{1}\right),\left(s_{2}, t_{2}\right) \in E\left(Q_{3}\right)$ or $\left(s_{1}, t_{1}\right),\left(s_{2}, t_{2}\right) \notin E\left(Q_{3}\right)$.

Proof. If $\left(s_{1}, t_{1}\right),\left(s_{2}, t_{2}\right) \in E\left(Q_{3}\right)$, then $Q_{3}$ can be divided into two $Q_{2}$ 's such that each subcube contains one source-sink pair. Each subcube is Hamiltonian-laceable, and thus there exists a 2-eq-DPC. If $\left(s_{1}, t_{1}\right),\left(s_{2}, t_{2}\right) \notin$ $E\left(Q_{3}\right)$, an arbitrary paired 2 -DPC is indeed a 2 -eq-DPC since the two paths in the 2-DPC are of length at least three and their length sum should be six. The existence of a paired 2-DPC is guaranteed by Lemma 1. Suppose $\left(s_{1}, t_{1}\right) \in E\left(Q_{3}\right)$ and $\left(s_{2}, t_{2}\right) \notin E\left(Q_{3}\right)$. It is straightforward to check that for any $s_{2}$ - $t_{2}$ path $P$ of length $3, Q_{3} \backslash V(P)$ is isomorphic to a path of length 3. Since $\left(s_{1}, t_{1}\right) \in E\left(Q_{3}\right)$, no 2-eq-DPC can be constructed. The proof is completed.

Lemma 6. Suppose that $S$ and $T$ are subsets of different parts of the bipartition of $Q_{3}$.

(a) If $\left(s_{1}, t_{1}\right) \in E\left(Q_{3}\right)$, there exists a paired 2-DPC joining $S$ and $T$ in which the $s_{1}-t_{1}$ path is of length 1 (and the $s_{2}-t_{2}$ path is of length 5) unless $\left\{s_{2}, t_{2}\right\}=V\left(Q_{3}\right) \backslash\left(N\left(s_{1}\right) \cup N\left(t_{1}\right)\right)$.

(b) Let $\left(s_{1}, t_{1}\right),\left(s_{2}, t_{2}\right) \in E\left(Q_{3}\right)$. If $\left(t_{1}, s_{2}\right) \in E\left(Q_{3}\right)$ or $\left(t_{2}, s_{1}\right) \in E\left(Q_{3}\right)$, there exist three kinds of paired 2-DPC's joining $S$ and $T$ such that the $s_{1}$ - $t_{1}$ path is of length 1,3 , and 5 , respectively.

Proof. Let $G^{\prime}$ be the subgraph of $Q_{3}$ induced by $V\left(Q_{3}\right) \backslash\left\{s_{1}, t_{1}\right\}$. If $\left(s_{1}, t_{1}\right) \in E\left(Q_{3}\right), G^{\prime}$ is isomorphic to the product of a path of length 1 
and a path of length 2. It is straightforward to check that for any balanced pair of vertices $s_{2}$ and $t_{2}, G^{\prime}$ has an $s_{2}-t_{2}$ Hamiltonian path if and only if $\left\{s_{2}, t_{2}\right\} \neq V\left(Q_{3}\right) \backslash\left(N\left(s_{1}\right) \cup N\left(t_{1}\right)\right)$. Thus, (a) is proved. The proof of (b) is a direct consequence of Lemmas 5 and 6(a).

Theorem 2. Every $m$-dimensional bipartite $H L$-graph $X_{m}, m \geq 4$, has a paired 2-eq-DPC joining $S$ and $T$ if $S$ and $T$ are subsets of different parts of the bipartition.

Proof. The proof is by an induction on $m$. Let $X_{m}=G_{0} \oplus G_{1}$, where each $G_{i}, i=0,1$, is isomorphic to an $X_{m-1}$. The proofs for the basis, i.e., when $m=4$, and the inductive step, i.e., when $m \geq 5$, are combined into one to avoid repetition. For the base case of $m=4$, we will use Lemmas 5 and 6 to find 2-DPC's in $G_{0}$ and $G_{1}$ that can be merged to obtain a desired 2-eq-DPC. For $m \geq 5$, we will assume that each $G_{i}, i=0,1$, has a 2 -eq-DPC joining balanced source-sink pairs. It is assumed w.l.o.g. that the number of terminals in $G_{0}$ is at least that in $G_{1}$. There are five cases depending on the positions of terminals.

Case 1: $s_{1}, t_{1} \in V\left(G_{0}\right)$ and $s_{2}, t_{2} \in V\left(G_{1}\right)$.

It suffices to construct an $s_{1}-t_{1}$ Hamiltonian path in $G_{0}$ and an $s_{2}$ - $t_{2}$ Hamiltonian path in $G_{1}$.

Case 2: $s_{1}, s_{2} \in V\left(G_{0}\right)$ and $t_{1}, t_{2} \in V\left(G_{1}\right)$.

If $m \geq 5$, we pick up arbitrary pseudo-terminals $t_{1}^{*}$ and $t_{2}^{*}$ and then find a 2-eq-DPC $\left[\left(s_{1}, t_{1}^{*}\right),\left(s_{2}, t_{2}^{*}\right) \mid G_{0}, \emptyset\right]$ and a 2 -eq-DPC $\left[\left(t_{1}^{*}, t_{1}\right),\left(t_{2}^{*}, t_{2}\right) \mid G_{1}, \emptyset\right]$. By merging them, we obtain a 2-eq-DPC in $X_{m}$. Let $m=4$. Note that picking up arbitrary pseudo-terminals $t_{1}^{*}$ and $t_{2}^{*}$ is not sufficient since each $G_{i}$ may not have a 2-eq-DPC even if all source-sink pairs are balanced. We pick up pseudo-terminals $t_{1}^{*}$ and $t_{2}^{*}$ from $N\left(s_{1}\right) \cap N\left(s_{2}\right)$. By Lemma 1, there

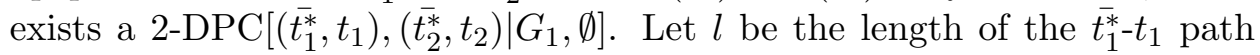
in the DPC. Then, $l \in\{1,3,5\}$. For any $l$, by Lemma 6 , there exists a 2 -DPC $\left[\left(s_{1}, t_{1}^{*}\right),\left(s_{2}, t_{2}^{*}\right) \mid G_{0}, \emptyset\right]$ in which the $s_{1}-t_{1}^{*}$ path is of length $6-l$. To obtain a 2-eq-DPC in $X_{m}$, it suffices to merge the two DPC's.

Case 3: $s_{1}, t_{2} \in V\left(G_{0}\right)$ and $t_{1}, s_{2} \in V\left(G_{1}\right)$.

In a similar manner to Case 2, a 2-eq-DPC joining $S$ and $T$ will be constructed. If $m \geq 5$, it suffices to pick up arbitrary pseudo-terminals $t_{1}^{*}$ and $s_{2}^{*}$, and then merge a 2 -eq-DPC $\left[\left(s_{1}, t_{1}^{*}\right),\left(s_{2}^{*}, t_{2}\right) \mid G_{0}, \emptyset\right]$ and a 2 -eq-DPC $\left[\left(\bar{t}_{1}^{*}, t_{1}\right)\right.$, $\left.\left(s_{2}, \bar{s}_{2}^{*}\right) \mid G_{1}, \emptyset\right]$. Let $m=4$. We select pseudo-terminals $t_{1}^{*}$ and $s_{2}^{*}$ so that there exist three kinds of $2-\mathrm{DPC}\left[\left(s_{1}, t_{1}^{*}\right),\left(s_{2}^{*}, t_{2}\right) \mid G_{0}, \emptyset\right]$ of Lemma $6(\mathrm{~b})$. We first pick up $t_{1}^{*}$ in $N\left(s_{1}\right)$. If $\left(s_{1}, t_{2}\right) \in E\left(G_{0}\right)$, then pick up $s_{2}^{*}$ in $N\left(t_{2}\right)$; otherwise, pick up $s_{2}^{*}$ in $N\left(t_{2}\right) \cap N\left(t_{1}^{*}\right)$. Finally, a $2-\operatorname{DPC}\left[\left(\overline{t_{1}^{*}}, t_{1}\right),\left(s_{2}, \overline{s_{2}^{*}}\right) \mid G_{1}, \emptyset\right]$ and one of the three 2-DPC's in $G_{0}$ are merged to obtain a 2-eq-DPC. 
Case 4: $s_{1}, t_{1}, s_{2} \in V\left(G_{0}\right)$ and $t_{2} \in V\left(G_{1}\right)$.

If $m \geq 5$, we find a 2 -eq-DPC $\left[\left(s_{1}, t_{1}\right),\left(s_{2}, t_{2}^{*}\right) \mid G_{0}, \emptyset\right]$ for an arbitrary pseudoterminal $t_{2}^{*}$. For some edge $(x, y)$ on the $s_{1}-t_{1}$ path such that $\bar{x}, \bar{y} \neq t_{2}$, we find a 2 -eq-DPC $\left[(\bar{x}, \bar{y}),\left(\overline{t_{2}^{*}}, t_{2}\right) \mid G_{1}, \emptyset\right]$. It suffices to merge the two DPC's. Let $m=4$. We pick up a pseudo-terminal $t_{2}^{*}$ in $N\left(s_{1}\right) \cap N\left(s_{2}\right)$. Thus, there exists a $2-\mathrm{DPC}\left[\left(s_{1}, t_{1}\right),\left(s_{2}, t_{2}^{*}\right) \mid G_{0}, \emptyset\right]$ in which the $s_{2}-t_{2}^{*}$ path is of length 1 by Lemma $6(\mathrm{a})$. If there exists an edge $(x, y)$ on the $s_{1}-t_{1}$ path such that $\bar{x}$ and $\bar{y}$ are free, $(\bar{x}, \bar{y}) \in E\left(G_{1}\right)$, and $\left\{t_{2}^{*}, t_{2}\right\} \neq V\left(G_{1}\right) \backslash(N(\bar{x}) \cup N(\bar{y}))$, then

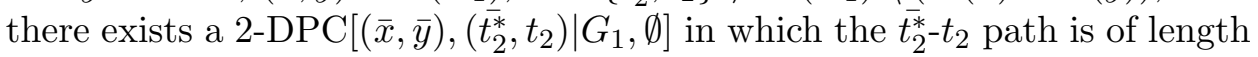
5 by Lemma $6(\mathrm{a})$. A 2-eq-DPC can be obtained by merging the two DPC's.

We must still show that such an edge $(x, y)$ exists. The $s_{1}-t_{1}$ path has a path segment $(u, v, w)$ such that $\bar{u}, \bar{v}, \bar{w} \neq t_{2}$. It suffices to prove a claim that for any path $P=(u, v, w)$ in $G_{0}$ and any $\alpha, \beta \in V\left(G_{1}\right)$ such that $c(\alpha) \neq c(\beta)$ and $\{\alpha, \beta\} \cap\{\bar{u}, \bar{v}, \bar{w}\}=\emptyset$, there exists an edge $(x, y)$ on $P$ such that (i) $(\bar{x}, \bar{y}) \in E\left(G_{1}\right)$ and (ii) $\{\alpha, \beta\} \neq V\left(G_{1}\right) \backslash(N(\bar{x}) \cup N(\bar{y}))$. Recall $m=4$. Note that for each vertex $p$ in $Q_{3}$, there is a unique vertex $q$ such that $c(q) \neq c(p)$ and $(q, p) \notin E\left(Q_{3}\right)$. Since $c(\bar{v}) \neq c(\bar{u})=c(\bar{w}),(\bar{u}, \bar{v})$ or $(\bar{v}, \bar{w})$ is an edge of $G_{1}$. If both $(\bar{u}, \bar{v})$ and $(\bar{v}, \bar{w})$ are edges of $G_{1}$, then at least one of $(u, v)$ and $(v, w)$ certainly satisfies (ii). Suppose that $(\bar{v}, \bar{w})$ is not an edge of $G_{1}$ and $(u, v)$ does not satisfy (ii). Since any vertex $z$ of $G_{1}$ such that $z \neq \bar{w}$ and $c(z)=c(\bar{w})$ is adjacent to $\bar{v}, \alpha$ or $\beta$ is adjacent to $\bar{v}$, which is a contradiction. Therefore, $(u, v)$ satisfies (ii). Hence, the claim is true.

Case 5: $s_{1}, t_{1}, s_{2}, t_{2} \in V\left(G_{0}\right)$.

If $m \geq 5$ or both $m=4$ and either $\left(s_{1}, t_{1}\right),\left(s_{2}, t_{2}\right) \in E\left(G_{0}\right)$ or $\left(s_{1}, t_{1}\right),\left(s_{2}, t_{2}\right) \notin$ $E\left(G_{0}\right)$, there exists a 2-eq-DPC $\left[\left(s_{1}, t_{1}\right),\left(s_{2}, t_{2}\right) \mid G_{0}, \emptyset\right]$ by induction hypothesis and Lemma 5. We claim that there exist two edges, $(u, v)$ on the $s_{1}-t_{1}$ path and $(x, y)$ on the $s_{2}$ - $t_{2}$ path such that a 2 -eq-DPC $\left[(\bar{u}, \bar{v}),(\bar{x}, \bar{y}) \mid G_{1}, \emptyset\right]$ exists. For $m \geq 5$, it is sufficient to pick up an arbitrary edge on each path. For $m=4$, due to the claim of Case 4 , there exist $(u, v)$ on the $s_{1}$ - $t_{1}$ path and $(x, y)$ on the $s_{2}-t_{2}$ path such that $(\bar{u}, \bar{v}),(\bar{x}, \bar{y}) \in E\left(G_{1}\right)$. Then, by Lemma 5 , the 2-eq-DPC in $G_{1}$ exists. It remains to merge the two 2-eq-DPC's.

Finally, let $m=4$ and $\left(s_{1}, t_{1}\right) \in E\left(G_{0}\right)$ and $\left(s_{2}, t_{2}\right) \notin E\left(G_{0}\right)$. By Lemma $6(\mathrm{a})$, there exists a $2-\mathrm{DPC}\left[\left(s_{1}, t_{1}\right),\left(s_{2}, t_{2}\right) \mid G_{0}, \emptyset\right]$ in which the $s_{1}-t_{1}$ path is of length 1 and the $s_{2}-t_{2}$ path is of length 5 . By the claim of Case 4 , there exists an edge $(x, y)$ on the $s_{2}$ - $t_{2}$ path such that $(\bar{x}, \bar{y}) \in E\left(G_{1}\right)$ and $\left\{\overline{s_{1}}, \overline{t_{1}}\right\} \neq V\left(G_{1}\right) \backslash(N(\bar{x}) \cup N(\bar{y}))$. Again by Lemma $6($ a $)$, there exists a 2 $\operatorname{DPC}\left[\left(\overline{s_{1}}, \overline{t_{1}}\right),(\bar{x}, \bar{y}) \mid G_{1}, \emptyset\right]$ in which the $\bar{x}-\bar{y}$ path is of length 1 and the $\overline{s_{1}}-\overline{t_{1}}$ path is of length 5. It suffices to merge the two DPC's. This completes the proof of Theorem 2 . 


\section{Strongly Hamiltonian-Laceability of Bipartite HL-Graphs}

In this section, it will be shown that if an $m$-dimensional bipartite-HL graph $X_{m}, m \geq 3$, has either at most $m-2$ faulty edges or one faulty vertex and at most $m-3$ faulty edges, the graph with the faulty elements removed is strongly Hamiltonian-laceable. Paired 2-disjoint path coverability of $X_{m}$ studied in the previous section will play an important role in the construction of an $L^{\mathrm{OPT}}$-path. We begin by considering strongly Hamiltonian-laceability of $Q_{3}$ having a unique vertex fault.

Lemma 7. For a faulty vertex $v_{f}$ in $Q_{3}, Q_{3} \backslash v_{f}$ is strongly Hamiltonianlaceable.

Proof. For a pair of fault-free vertices $s$ and $t$ with $c(s)=c(t) \neq c\left(v_{f}\right)$, an $L^{\mathrm{OPT}}$-path joining them exists by Lemma 3. Now, at least one of $s$ and $t$ has the same color as $v_{f}$. There exist three induced subgraphs of $Q_{3} \backslash v_{f}$ that are isomorphic to the product of a path of length 1 and a path of length 2 . Among the three, at least two contain both $s$ and $t$. It is immediately seen that at least one has an $L^{\mathrm{OPT}}$-path joining $s$ and $t$. The $L^{\mathrm{OPT}}$-path is indeed an $L^{\mathrm{OPT}}$-path of $Q_{3} \backslash v_{f}$. Therefore, the lemma is proved.

Theorem 3. Let $X_{m}$ be an $m$-dimensional bipartite $H L$-graph. For $m \geq 3$, (a) $X_{m}$ is $(m-2)$-edge-fault strongly Hamiltonian-laceable, and (b) $X_{m}$ is 1-vertex-fault and $(m-3)$-edge-fault strongly Hamiltonian-laceable.

Proof. The proof is by induction on $m$. Let $X_{m}=G_{0} \oplus G_{1}$, where each $G_{i}, i=0,1$, is isomorphic to an $(m-1)$-dimensional bipartite HL-graph. For any fault-free vertices $s$ and $t$, an $L^{\mathrm{OPT}}$-path in $X_{m}$ joining them will be constructed. Since an $L^{\mathrm{OPT}}$-path in $X_{m}$ with a virtual fault set $F \cup F^{\prime}$, where $F^{\prime}$ is a set of arbitrary $m-2-|F|$ fault-free edges, is also an $L^{\mathrm{OPT}}$-path in $X_{m}$ with the fault set $F$, we can assume $|F|=m-2$.

To prove (a), it is sufficient to consider the case when $c(s)=c(t)$ due to Lemma 2. Let $(x, y)$ be an arbitrary faulty edge such that $c(x) \neq c(s)=c(t)$. Observe that an $L^{\mathrm{OPT}}$-path joining $s$ and $t$ in $X_{m} \backslash F^{\prime}$, where $F^{\prime}=F \cup\{x\} \backslash$ $(x, y)$, is also an $L^{\mathrm{OPT}}$-path joining $s$ and $t$ in $X_{m} \backslash F$. This implies that it suffices to prove that $X_{m}$ with one faulty vertex and $m-3$ faulty edges is strongly Hamiltonian-laceable, which corresponds to the (b) of Theorem 3.

Let us begin by proving (b). The base case when $m=3$ is shown in Lemma 7. Let $m \geq 4$. It is assumed w.l.o.g. that the unique faulty vertex $v_{f}$ is contained in $G_{0}$, so $\left|F_{1}\right| \leq m-3$. There are three cases depending on the positions of $s$ and $t$. To construct an $L^{\mathrm{OPT}}$-path joining $s$ and $t$, in 


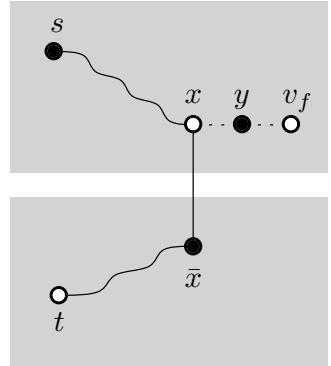

(a) Case 2

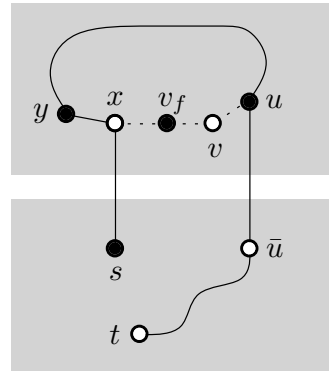

(b) Case $3,\left|F_{0}\right|=m-2$

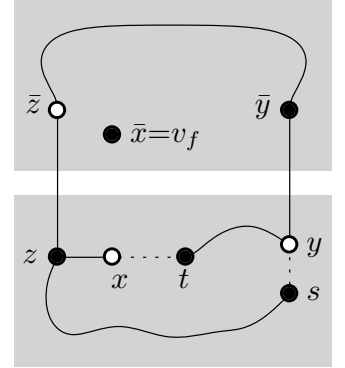

(c) Case $3,\left|F_{1}\right|=m-3$

Figure 3: Illustration of the proof of the Theorem 3.

almost all the cases, we merge an $L^{\mathrm{OPT}}$-path of $G_{0}$ and either a Hamiltonian path or a paired 2-DPC of $G_{1}$. It is straightforward to see that the resultant path is always an $L^{\mathrm{OPT}}$-path since a Hamiltonian path or a paired 2-DPC of $G_{1}$ covers all the vertices of $G_{1}$. Note that merging two $L^{\mathrm{OPT}}$-paths, one in each $G_{i}$, may not result in an $L^{\mathrm{OPT}}$-path of $X_{m}$.

Case 1: $s, t \in V\left(G_{0}\right)$.

We let $F_{0}^{\prime}=F_{0}$ if $\left|F_{0}\right| \leq m-3$; otherwise, let $F_{0}^{\prime}=F_{0} \backslash e$ for an arbitrary faulty edge $e$ in $G_{0}$. Then, $\left|F_{0}^{\prime}\right| \leq m-3$. There exists an $L^{\mathrm{OPT}}$-path $P_{0}$ in $G_{0} \backslash F_{0}^{\prime}$ joining $s$ and $t$ by induction hypothesis. If $P_{0}$ passes through the faulty edge $e$, let $(u, v)$ be the faulty edge; otherwise, let $(u, v)$ be an edge on $P_{0}$ such that $(u, \bar{u})$ and $(v, \bar{v})$ are fault-free. Such an edge $(u, v)$ exists since $\left\lfloor\left(2^{m-1}-1\right) / 2\right\rfloor>m-3$ for every $m \geq 4$. To construct a desired $L^{\mathrm{OPT}}$-path of $X_{m}$, it suffices to replace $(u, v)$ with a path $\left(u, P_{1}, v\right)$, where $P_{1}$ is an $\bar{u}-\bar{v}$ Hamiltonian path in $G_{1}$.

Case 2: $s \in V\left(G_{0}\right)$ and $t \in V\left(G_{1}\right)$.

If $\left|F_{0}\right| \leq m-3$, we first pick up a pseudo-terminal $t^{*}$. There exist an $L^{\mathrm{OPT}}$ path joining $s$ and $t^{*}$ in $G_{0}$ and a $t^{*}-t$ Hamiltonian path in $G_{1}$. Merging these paths results in a desired $L^{\mathrm{OPT}}$-path. Let $\left|F_{0}\right|=m-2$. (See Fig. 3a.) Regarding $v_{f}$ as a virtual fault-free vertex, we find an $L^{\mathrm{OPT}}$-path $P_{0}$ joining $s$ and $v_{f}$ in $G_{0} \backslash\left(F_{0} \backslash v_{f}\right)$. Let $P_{0}=\left(s, P_{x}, x, y, v_{f}\right)$ for some path segment $P_{x}$. Notice that $\left(s, P_{x}, x, y\right)$ and $\left(s, P_{x}, x\right)$ are $L^{\mathrm{OPT}}$-paths in $G_{0} \backslash F_{0}$. If $c(t)=c(y)$, a desired $L^{\mathrm{OPT}}$-path is obtained by merging $\left(s, P_{x}, x, y\right)$ and a $\bar{y}$ - $t$ Hamiltonian path in $G_{1}$; otherwise it is obtained by merging $\left(s, P_{x}, x\right)$ and an $\bar{x}-t$ Hamiltonian path in $G_{1}$.

Case 3: $s, t \in V\left(G_{1}\right)$.

If $\left|F_{0}\right| \leq m-3$ and $\left|F_{1}\right| \leq m-4$, we pick up two pseudo-terminals $s^{*}$ and $t^{*}$. To obtain a desired $L^{\mathrm{OPT}}$-path, it suffices to merge an $L^{\mathrm{OPT}}$ - 
path joining $s^{*}$ and $t^{*}$ in $G_{0} \backslash F_{0}$ and a $2-\operatorname{DPC}\left[\left(s, \bar{s}^{*}\right),\left(\bar{t}^{*}, t\right) \mid G_{1}, F_{1}\right]$. The existence of the 2-DPC is due to Theorem 1 and Lemma 1 . Now, let $\left|F_{0}\right|=m-2$ and $\left|F_{1}\right|=\left|F_{2}\right|=0$ for the second case. (See Fig. 3b.) Regarding $v_{f}$ as a virtual free vertex, we can find a Hamiltonian cycle $C=\left(x, y, P_{y}, u, v, v_{f}\right)$. The Hamiltonian cycle exists since $G_{0}$ is $(m-3)$ edge-fault strongly Hamiltonian-laceable. If we remove $v_{f}$ from $C$, we have a Hamiltonian path $P_{0}=\left(x, y, P_{y}, u, v\right)$ of $G_{0} \backslash F_{0}$. Note that $c(x)=$ $c(v) \neq c\left(v_{f}\right)$ and $c(y)=c(u)=c\left(v_{f}\right)$. It follows that $P_{x, v}=\left(x, y, P_{y}, u, v\right)$, $P_{x, u}=\left(x, y, P_{y}, u\right), P_{y, v}=\left(y, P_{y}, u, v\right)$, and $P_{y, u}=\left(y, P_{y}, u\right)$ are all $L^{\mathrm{OPT}_{-}}$ paths in $G_{0} \backslash F_{0}$. When $c(s)=c(t) \neq c\left(v_{f}\right)$, a desired $L^{\mathrm{OPT}}$-path is obtained by merging $P_{x, v}$ and a $2-\operatorname{DPC}\left[(s, \bar{x}),(\bar{v}, t) \mid G_{1}, \emptyset\right]$. When $c(s)=c(t)=c\left(v_{f}\right)$, a desired $L^{\mathrm{OPT}}$-path is obtained from $P_{y, u}$ and a 2 -DPC $\left[(s, \bar{y}),(\bar{u}, t) \mid G_{1}, \emptyset\right]$. Let $c(s) \neq c(t)$. We assume w.l.o.g. $c(s)=c\left(v_{f}\right)$. At least one of $\{\bar{x}, \bar{u}\}$ and $\{\bar{y}, \bar{v}\}$ is different from $\{s, t\}$, say $\{\bar{x}, \bar{u}\} \neq\{s, t\}$. If $\{\bar{x}, \bar{u}\} \cap\{s, t\}=\emptyset$, a desired $L^{\mathrm{OPT}}$-path is obtained from $P_{x, u}$ and a $2-\operatorname{DPC}\left[(s, \bar{u}),(\bar{x}, t) \mid G_{1}, \emptyset\right]$. If $\{\bar{x}, \bar{u}\} \cap\{s, t\} \neq \emptyset$, then we have either $\bar{x}=s$ or $\bar{u}=t$, say $\bar{x}=s$. Then, a desired $L^{\mathrm{OPT}}$-path is obtained from $P_{x, u}$ and a Hamiltonian path joining $\bar{u}$ and $t$ in $G_{1} \backslash s$.

Finally, let $\left|F_{1}\right|=m-3, F_{0}=\left\{v_{f}\right\}$, and $F_{2}=\emptyset$ for the last case. If $c(s) \neq c(t)$, we find a Hamiltonian path joining $s$ and $t$ in $G_{1} \backslash F_{1}$, and then for some edge $(u, v)$ on the Hamiltonian path such that $\bar{u}, \bar{v} \neq v_{f}$, the edge $(u, v)$ is replaced with $\left(u, P_{0}, v\right)$, where $P_{0}$ is an $L^{\mathrm{OPT}}$-path joining $\bar{u}$ and $\bar{v}$ in $G_{0} \backslash F_{0}$. The path obtained is a desired $L^{\mathrm{OPT}}$-path. Now, assume that $c(s)=c(t)$. (See Fig. 3c.) There exists a Hamiltonian cycle $C$ in $G_{1} \backslash F_{1}$. Let $C=\left(s, P_{x}, x, t, P_{y}, y\right)$ for some path segments $P_{x}$ and $P_{y}$, which are possibly paths of length 0 . Then, we have two paths $P_{s}=\left(s, P_{x}, x\right)$ and $P_{t}=\left(t, P_{y}, y\right)$ that cover all the vertices in $G_{1}$. If $v_{f} \notin\{\bar{x}, \bar{y}\}$, a desired $L^{\mathrm{OPT}}$-path is obtained from $P_{s}, P_{t}$, and an $L^{\mathrm{OPT}}$-path in $G_{0} \backslash F_{0}$ joining $\bar{x}$ and $\bar{y}$. Suppose $v_{f} \in\{\bar{x}, \bar{y}\}$. Then, we have $c(s)=c(t)=c\left(v_{f}\right)$. Note that in this subcase, any $L^{\mathrm{OPT}}$-path leaves out two vertices having colors different from $v_{f}$. We assume $v_{f}=\bar{x}$, and let $P_{x}=\left(P_{z}, z\right)$ so $P_{s}=\left(s, P_{z}, z, x\right)$; or $P_{s}=(s=z, x)$ if $P_{x}$ is of length 0 . An $L^{\mathrm{OPT}}$-path joining $s$ and $t$ that does not contain $x$ is obtained from $P_{s} \backslash x, P_{t}$, and an $L^{\mathrm{OPT}}$-path in $G_{0} \backslash F_{0}$ joining $\bar{z}$ and $\bar{y}$. This completes the proof of Theorem 3 .

Theorem 3 is an extension of the work in $[21,26]$, which is stated in Lemma 2. The total number $m-2$ of faulty elements in Theorem 3 is the maximum possible regardless of whether there exists a faulty vertex or not. This can be verified as follows. Suppose that $s$ and $t$ are two vertices of $X_{m}$ that are adjacent via a fault-free edge. If there are $m-1$ faulty edges 
incident to $s$ or $m-2$ faulty edges incident to $s$ and one faulty vertex $v_{f}$ adjacent to $s$, then there exists no $L^{\mathrm{OPT}}$-path joining $s$ and $t$.

\section{Conclusions}

In this paper, we investigated 2-disjoint path cover problems as an early stage in bipartite HL-graph research. It was proved that every $X_{m}, m \geq 4$, with $m-3$ or less faulty edges has a paired 2-DPC joining $S$ and $T$ provided $S \cup T$ is balanced. We also showed that every $X_{m}, m \geq 4$, has a paired 2eq-DPC joining $S$ and $T$ if $S$ and $T$ are subsets of different parts of the bipartition. Using 2-DPC properties, it was proved that every $X_{m}, m \geq 3$, is $(m-2)$-edge-fault strongly Hamiltonian-laceable and is 1-vertex-fault and $(m-3)$-edge-fault strongly Hamiltonian-laceable. Both the number $m-3$ of allowed faulty edges for the paired 2-DPC and the number $m-2$ of faulty elements for the strongly Hamiltonian-laceability are the maximum possible.

As a result, a balanced pair of vertices $s$ and $t$ of $X_{m}, m \geq 4$, with $m-3$ or less faulty edges are joined by a Hamiltonian path passing through an arbitrary prescribed edge $(x, y)$ such that $\{x, y\} \neq\{s, t\}$ (by Theorem 1 when $|\{x, y\} \cap\{s, t\}|=0$ and by Theorem 3(b) when $|\{x, y\} \cap\{s, t\}|=1$ ). We conjecture that for some constant $c \geq 4$, every $X_{m}, m \geq c$, possessing $f$ or less faulty elements (vertices and/or edges) has a paired $k$-DPC for any $f$ and $k \geq 2$ with $f+2 k \leq m+1$ under the 'balancedness' condition of $k^{b}+2 f_{v}^{b}=k^{w}+2 f_{v}^{w}$, where $k^{b}$ and $k^{w}$ respectively are the numbers of black and white terminals and $f_{v}^{b}$ and $f_{v}^{w}$ respectively are the numbers of black and white vertex faults. It will be a challenging problem to verify this conjecture.

\section{Acknowledgement}

The authors are very grateful to the anonymous reviewers for their comments and suggestions. This research was supported by Basic Science Research Program through the National Research Foundation of Korea (NRF) funded by the Ministry of Education, Science and Technology (grant number 2010-0010626). This work was also supported by the Catholic University of Korea, Research Fund, 2011.

\section{References}

[1] K. Asdre and S.D. Nikolopoulos, "The 1-fixed-endpoint path cover problem is polynomial on interval graphs," Algorithmica 58(3), pp. 679-710, 2010. 
[2] J.A. Bondy and U.S.R. Murty, Graph Theory, 2nd printing, Springer, 2008.

[3] X.-B. Chen, "Many-to-many disjoint paths in faulty hypercubes," Information Sciences 179(18) (2009) 3110-3115.

[4] X.-B. Chen, "Paired many-to-many disjoint path covers of the hypercubes," Information Sciences (2013), <doi:http://dx.doi.org/10. 1016/j.ins. 2013.02.028>.

[5] P. Cull and S.M. Larson, "The Möbius cubes," in Proc. of the 6th IEEE Distributed Memory Computing Conf. (1991) 699-702.

[6] T. Dvořák and P. Gregor, "Hamiltonian paths with prescribed edges in hypercubes," Discrete Mathematics 307(16) (2007) 1982-1998.

[7] T. Dvořák and P. Gregor, "Partitions of faulty hypercubes into paths with prescribed endvertices," SIAM Journal on Discrete Mathematics 22(4) (2008) 1448-1461.

[8] K. Efe, "The crossed cube architecture for parallel computation," IEEE Trans. on Parallel and Distributed Systems 3(5) (1992) 513-524.

[9] J. Fink and P. Gregor, "Long paths and cycles in hypercubes with faulty vertices," Information Sciences 179(20) (2009) 3634-3644.

[10] J.-S. Fu, "Longest fault-free paths in hypercubes with vertex faults," Information Sciences 176(7) (2006) 759-771.

[11] P. Gregor and T. Dvořák, "Path partitions of hypercubes," Inf. Process. Lett. 108(6) (2008) 402-406.

[12] P.A.J. Hilbers, M.R.J. Koopman, and J.L.A. van de Snepscheut, "The Twisted Cube," in: J. Bakker, A. Nijman, P. Treleaven, eds., PARLE: Parallel Architectures and Languages Europe, Vol. I: Parallel Architectures, Springer (1987) 152-159.

[13] L.-H. Hsu and C.-K. Lin, Graph Theory and Interconnection Networks, CRC Press, 2008.

[14] C.-N. Hung and K.-C. Hu, "Fault-tolerant Hamiltonian laceability of bipartite hypercube-like networks," in: International Computer Symposium (2004) 1145-1149. 
[15] S.-Y. Kim and J.-H. Park, "Paired many-to-many disjoint path covers in recursive circulants $G\left(2^{m}, 4\right)$," IEEE Trans. on Computers (2012), <http://doi.ieeecomputersociety.org/10.1109/TC.2012.133>.

[16] Y. Kobayashi and C. Sommer, "On shortest disjoint paths in planar graphs," Discrete Optimization 7(4) (2010) 234-245.

[17] T.-L. Kueng, T. Liang, L.-H. Hsu, and J.J.M. Tan, "Long paths in hypercubes with conditional node-faults," Information Sciences 179(5) (2009) 667-681.

[18] C.-N. Lai, "Two conditions for reducing the maximal length of nodedisjoint paths in hypercubes," Theoretical Computer Science 418 (2012) 82-91.

[19] P.-L. Lai and H.-C. Hsu, "The two-equal-disjoint path cover problem of matching composition network," Inf. Process. Lett. 107(1) (2008) $18-23$.

[20] H.-S. Lim, J.-H. Park, and H.-C. Kim, "The bicube: an interconnection of two hypercubes," manuscript, 2010.

[21] C.-K. Lin, T.-Y. Ho, J.J.M. Tan, and L.-H. Hsu, "Fault-tolerant Hamiltonian laceability and fault-tolerant conditional Hamiltonian for bipartite hypercube-like networks," Journal of Interconnection Networks 10 (2009) 243-251.

[22] C.-K. Lin, J.J.M. Tan, D.F. Hsu, and L.-H. Hsu, "On the spanning connectivity and spanning laceability of hypercube-like networks," Theoretical Computer Science 381(1-3) (2007) 218-229.

[23] T.-C. Lin and D.-R. Duh, "Constructing vertex-disjoint paths in $(n, k)$ star graphs," Information Sciences 178(3) (2008) 788-801.

[24] J.A.M. McHugh, Algorithmic Graph Theory, Prentice-Hall, Englewood Cliffs, NJ, 1990.

[25] S.C. Ntafos and S.L. Hakimi, "On path cover problems in digraphs and applications to program testing," IEEE Trans. Software Eng. 5(5), pp. 520-529, Sept. 1979.

[26] C.-D. Park, Topological Properties of the Class of Hypercube-Like Graphs, Ph.D. Thesis, KAIST, 2004. 
[27] C.-D. Park and K.-Y. Chwa, "Hamiltonian properties on the class of hypercube-like networks," Inf. Process. Lett. 91(1) (2004) 11-17.

[28] J.-H. Park, H.-C. Kim, and H.-S. Lim, "Fault-Hamiltonicity of hypercube-like interconnection networks," in: Proc. 19th IEEE International Parallel and Distributed Processing Symposium (IPDPS'05), 2005.

[29] J.-H. Park, H.-C. Kim, and H.-S. Lim, "Many-to-many disjoint path covers in hypercube-like interconnection networks with faulty elements," IEEE Trans. on Parallel Distrib. Syst. 17(3) (2006) 227-240.

[30] J.-H. Park, H.-C. Kim, and H.-S. Lim, "Many-to-many disjoint path covers in the presence of faulty elements," IEEE Trans. on Computers 58(4) (2009) 528-540.

[31] A.S. Vaidya, P.S.N. Rao, and S.R. Shankar, "A class of hypercube-like networks," in: Proc. Fifth IEEE Symposium on Parallel and Distributed Processing (1993) 800-803.

[32] S. Wang, J. Li, and R. Wang, "Hamiltonian paths and cycles with prescribed edges in the 3 -ary $n$-cube," Information Sciences 181(14) (2011) 3054-3065. 